\title{
Estimated Performance of UWB Impulse Radio Transmission Including Dirty RF Effects
}

\author{
Institut für Höchstfrequenztechnik und Elektronik (IHE) \\ Universität Karlsruhe (TH), Germany \\ Tel: +49-721-608-6255, Fax: +49-721-691-865 \\ E-mail: jens.timmermann@ihe.uka.de
}

Jens Timmermann, Student Member, IEEE, Elena Pancera, Student Member, IEEE, Grzegorz Adamiuk, Student Member, IEEE, Werner Wiesbeck, Fellow, IEEE, and Thomas Zwick, Senior Member, IEEE

\begin{abstract}
For studying the performance of an ultra-wideband (UWB) system, either measurements or simulations can be performed. Simulations for performance predictions are more flexible, but they require an accurate system modeling that takes into account the non-ideal hardware. This paper presents the UWB indoor channel modeling, including relevant, non-ideal hardware components for the UWB impulse radio technique. As a performance measure the estimated bit error rate versus distance and versus signal-to-noise ratio for Time Hopping Pulse Position Modulation (TH-PPM) in a Line of Sight indoor scenario is presented. The influence of the data rate on the bit error rate is investigated as well.
\end{abstract}

Keywords - UWB, TH-PPM, impulse radio, non-ideal components, frontend modeling, bit error rate

\section{INTRODUCTION}

According to Shannon's law, capacity and hence data rate increases linearly with bandwidth, which makes the ultrawideband (UWB) technology a very promising future candidate for the transmission of ultra high data rates. Up to now, only few UWB systems are commercially available, and ultra-wideband transmission is still a topic of research. An important question is which system performance can be achieved in reality using UWB techniques. For example, the bit error rate $(B E R)$ at a given data rate is a topic of interest. Of course, the answer depends on the system architecture and on the hardware components that are always non-ideal in some sense. Whenever a prototype or an existing system is available, it is straight forward to study the performance by measurements. In many cases however, engineers are interested in trade off studies and the performance before or during the development stage of a hardware system, which results in the problem to know the performance. As a consequence, the system has to be modeled carefully, to enable performance simulations.

In this paper, a simulation-based approach, as an extension of [1], is presented for the UWB impulse radio transmission, a technique which does not use a carrier for transmission. Section II describes the system model and the component modeling. Especially the non-ideal characteristics of the components, which are experienced in reality and which lead to so called Dirty RF [2] effects are taken into consideration. The models are either based on measurement data of real components or on simulation methods that are extended to the ultra-wideband case. Section III presents the estimated performance of the non-ideal impulse radio system in terms of the bit error rate. The BER is determined as a function of distance, as well as a function of the signal-to-noise-ratio (SNR). Furthermore, the influence of the pulse repetition frequency (PRF) on the BER is analyzed. Finally, a conclusion is given in section IV.

\section{SYSTEM MODEL}

Fig. 1 shows the system model for the analysed impulse radio UWB transmission.

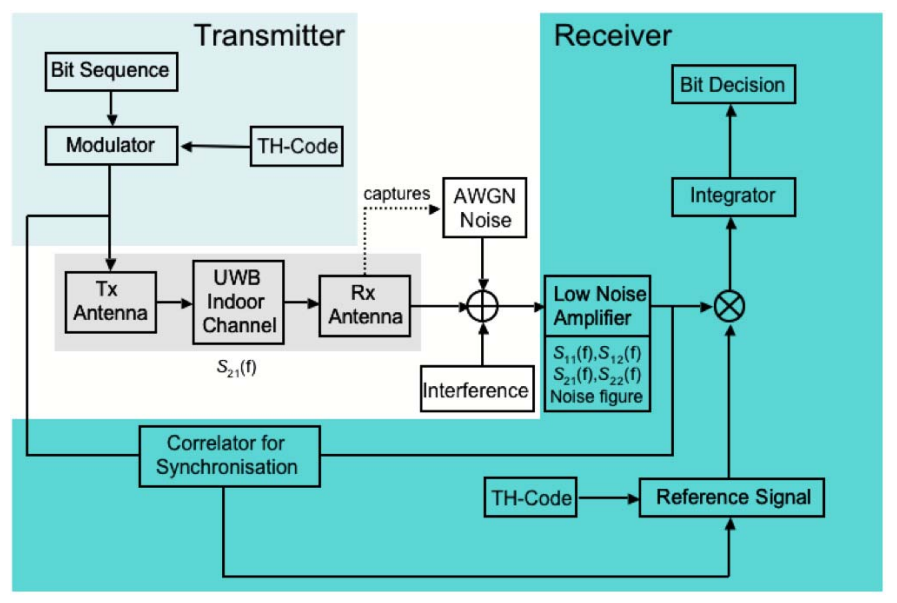

Fig. 1 System model of non-ideal UWB transmission

The transmitter (Tx) consists of an oscillator that generates the pulse repetition frequency, a modulator which includes time hopping $(\mathrm{TH})$ coding to separate users in a multi user scenario and the transmit antenna which radiates the signal over an indoor channel to the receive antenna. At the receiver (Rx) side the Rx antenna is followed by an UWB low noise amplifier (LNA). To reconstruct the bit sequence, the signal is multiplied by a template signal using the same pulse repetition frequency and the same TH code, and finally, the product is integrated over the bit duration and compared to a threshold for bit decision.

To study the behavior of a carrierless UWB system by a simulation-based approach, the single elements have to be modeled accurately. Taking into account Dirty RF effects means that non-ideal hardware and interference from other 
systems/transmitters operating in the considered frequency range have to be regarded as well. Therefore, the above mentioned system components are implemented as non-ideal, including noise. Impacts from other transmitters are modeled by the component "interference".

In this paper, the following non-ideal components and effects are considered: antennas, multipath indoor UWB channel, noise, interference, and a low noise amplifier at the receiver side. Since antenna characteristics depend on frequency, the antenna model of both antennas, Tx and Rx, respectively, is implemented by the antenna's complex 3D patterns. These are measured at 1601 frequencies within the frequency range from $2.5 \mathrm{GHz}$ to $12.5 \mathrm{GHz}$. The model for the indoor propagation channel is based on Ray Tracing. For given $\mathrm{Tx}$ and $\mathrm{Rx}$ positions, simulations are performed for the same set of frequencies like above. For each frequency, the paths are weighted by the transmitter's and the receiver's antenna patterns before they are coherently superposed. The result is the complex transfer function $S_{21}(f)$, and due to reciprocity $S_{12}(f)=S_{21}(f)$. For simplification, perfect matching of the antennas is assumed, but necessarily required for the simulation. The receive antenna is modeled like the transmit antenna, but since the receive antenna also captures thermal noise, a Additive White Gaussian Noise (AWGN) is added to the receive path. Since an indoor scenario is considered, the noise temperature of the receive antenna is assumed to have room temperature at $300 \mathrm{~K}$. Narrow band and wideband interference is added. It can represent for example UWB interference, caused by other users in a multi-user UWB scenario. Narrow band interference may for example represent WLAN interference at $5 \mathrm{GHz}$.

The low noise amplifier is described by the:

- measured frequency dependent $S$-parameters

- noise figure

$-1 \mathrm{~dB}$ compression point

- saturation power

- third order intercept point at the center frequency

This means that for simplification, the frequency dependence of these measures is neglected and matching is assumed.

\section{SYSTEM SIMULATION}

For simulation, system components and system parameters are defined: The pulse repetition frequency $P R F$ is $333 \mathrm{MHz}$ and the number of pulses per bit $N$ is 2 . The bit rate can be determined by Eq. (1):

$$
R=\frac{1 b i t}{N \cdot 1 / P R F}=\frac{1 b i t}{N} \cdot P R F
$$

For the chosen parameters Eq. (1) results in a bit rate of 166.67 Mbit/s. The chosen pulse shape is the second derivative of a Gaussian mono-pulse with a pulse width of 100 ps. The applied modulation technique is pulse position modulation (PPM). To separate users in a multi-user scenario, a random $\mathrm{TH}$ code is implemented. The modulated and coded signal has a Tx power $P_{\mathrm{Tx}}=-16.7 \mathrm{dBm}$ within the frequency range from $3.1 \mathrm{GHz}$ to $10.6 \mathrm{GHz}$ which is about $14 \mathrm{~dB}$ less compared to the maximal allowed $P_{\text {Txmax }}=-2.52 \mathrm{dBm}$ within the FCC mask. For the channel a typical laboratory indoor scenario as shown in Fig. 2 is chosen, with tables, instruments and shelves. While the Tx position is fixed, different $\mathrm{Rx}$ positions with different Tx - Rx distance are simulated. Spatial averaging of the signal around a given $\mathrm{Rx}$ position is not applied, since fading of the UWB signal is assumed to be neglectable according to the results shown in [4]. The antennas are a mono-cone $\mathrm{Tx}$ antenna and a mono-pole $\mathrm{Rx}$ antenna. The $\mathrm{Rx}$ noise temperature of $300 \mathrm{~K}$ results in an additive noise power of $P_{\mathrm{N}}=-75.2 \mathrm{dBm}$ within $3.1 \mathrm{GHz}$ to $10.6 \mathrm{GHz}$. The UWB interference is added with a constant power spectral density (PSD) versus frequency, representing other active UWB users. Its integral level $P_{\mathrm{PSD}}$ can be swept in order to simulate different signal-to-noiseratios at a fixed distance. The power level is chosen in steps of $5 \mathrm{~dB}$ in the range $-77.125 \mathrm{dBm}<P_{\mathrm{PSD}}<-52.125 \mathrm{dBm}$.

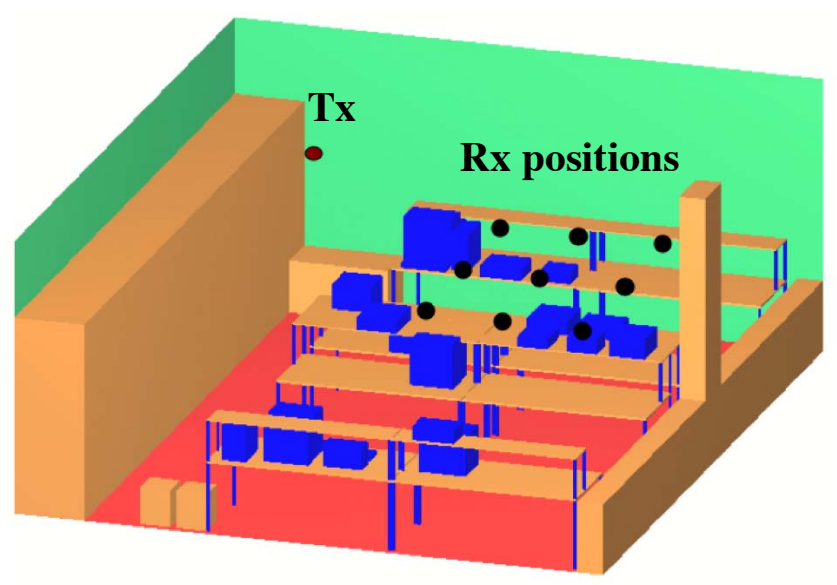

Fig. 2 Indoor environment together with transmitter and receiver positions

The low noise amplifier (HMC-C022) is modeled with a gain of $G=14-15 \mathrm{~dB}$ within the FCC mask and a noise figure $N F=2.5 \mathrm{~dB}$ at $8 \mathrm{GHz}$. Fig. 3 shows the simulated bit error rate $B E R$ versus the $\mathrm{Tx}-\mathrm{Rx}$ distance. Each curve is based on five $\mathrm{Rx}$ positions (five distances).

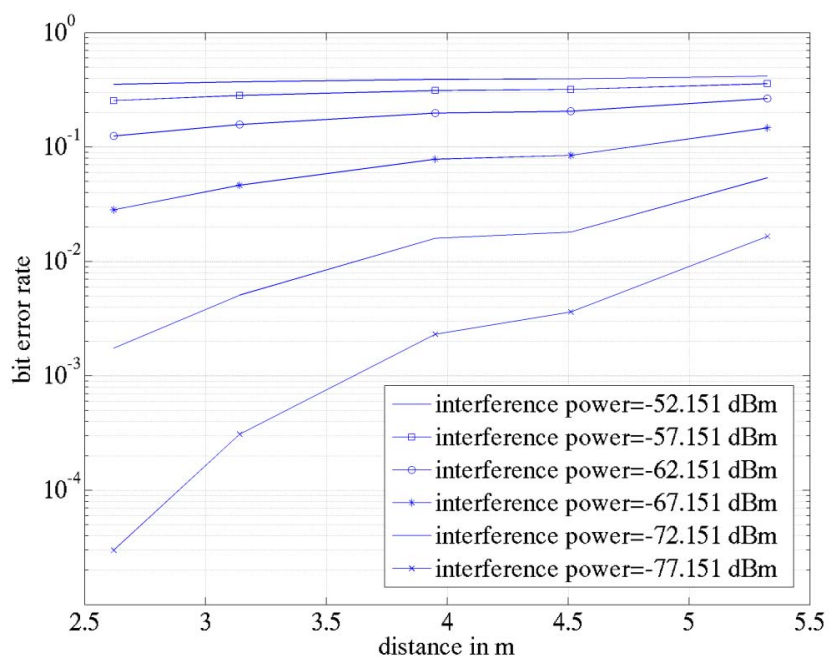

Fig. 3 Bit error rate versus distance for different levels of interference power for a bit rate of $166.67 \mathrm{Mbit} / \mathrm{s}$ 
Six different levels of interference power $P_{\mathrm{PSD}}$ are simulated, which leads to six curves. For a fixed distance, the $B E R$ degrades with increasing interference power. As the distance increases, the $B E R$ gets worse and tends to 0.5 . At given distance it also tends to 0.5 for very high interference levels. The results make sense and demonstrate, which bit error rates can be achieved for a bit rate of $166.67 \mathrm{Mbit} / \mathrm{s}$ in an indoor Line of Sight scenario taking into account non-ideal components.

The same simulation data is visualized in terms of $B E R$ versus $S N R$ in Fig. 4, where the $S N R$ includes the contribution from the interference. For each distance, the interference power is swept, leading to a $B E R-S N R$ curve. For the simulations it is assumed that the received power $P_{\mathrm{Rx}}$ at the $\mathrm{Rx}$ antenna output represents the signal power. In Fig. 4 all five curves represent the same BER-SNR behavior of the system, which can be approximated by a complementary error function that leads to a $B E R$ of 0.5 for very small $S N R$.

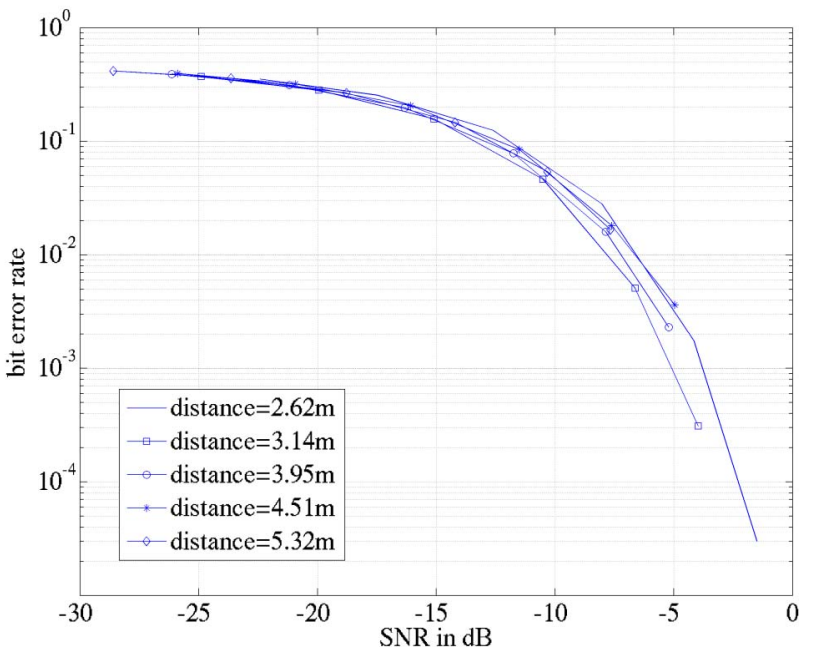

Fig. 4 Bit error rate versus signal-to-noise-ratio for a bit rate of $166.67 \mathrm{Mbit} / \mathrm{s}$

Fig. 3 and 4 represent the system behavior for a bit rate of 166.67 MBit/s $(P R F=333 \mathrm{MHz}, N=2)$. An interesting question is: what happens if the bit rate is varied by varying only the PRF?

For comparison, besides the bit rate of $166.67 \mathrm{Mbit} / \mathrm{s}$, a reduced bit rate of $55.55 \mathrm{Mbit} / \mathrm{s}$ is simulated which corresponds to a PRF of $111.11 \mathrm{MHz}$. The number of pulses $N$ per bits is 2 and not changed. The dependence of the $B E R$ on distance for the reduced bit rate of $55.55 \mathrm{Mbit} / \mathrm{s}$ can be seen in Fig. 5.

Comparing Fig. 5 and Fig. 3, the following conclusion can be drawn: As the bit rate is reduced, the $B E R$ becomes better, but this improvement is only small and can only be seen in the figures for good $B E R$ values. This can be explained by the fact that an increase of pulse repetition time leads to less influence of multi path components.

Fig. 6 presents the $B E R$ versus $S N R$ for the reduced bit rate of $55.55 \mathrm{Mbit} / \mathrm{s}$. Again, all curves combine almost to one single curve like in Fig. 4, but comparing Fig. 6 and Fig. 4, the following conclusion can be drawn: For a given $S N R$, the $B E R$ resulting from the reduced bit rate is better.

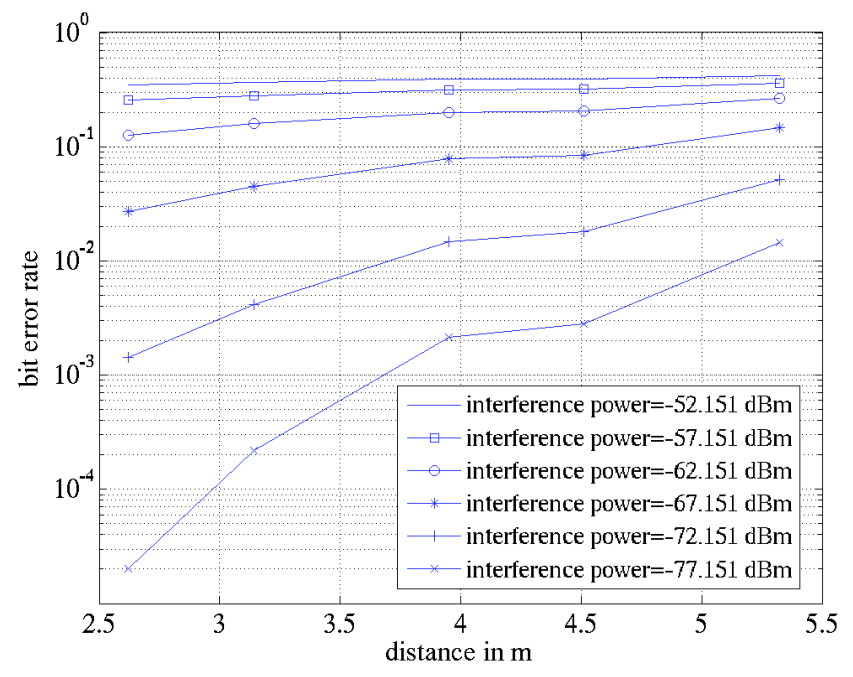

Fig. 5 Bit error rate versus distance for different levels of interference power for a bit rate of $55.55 \mathrm{Mbit} / \mathrm{s}$

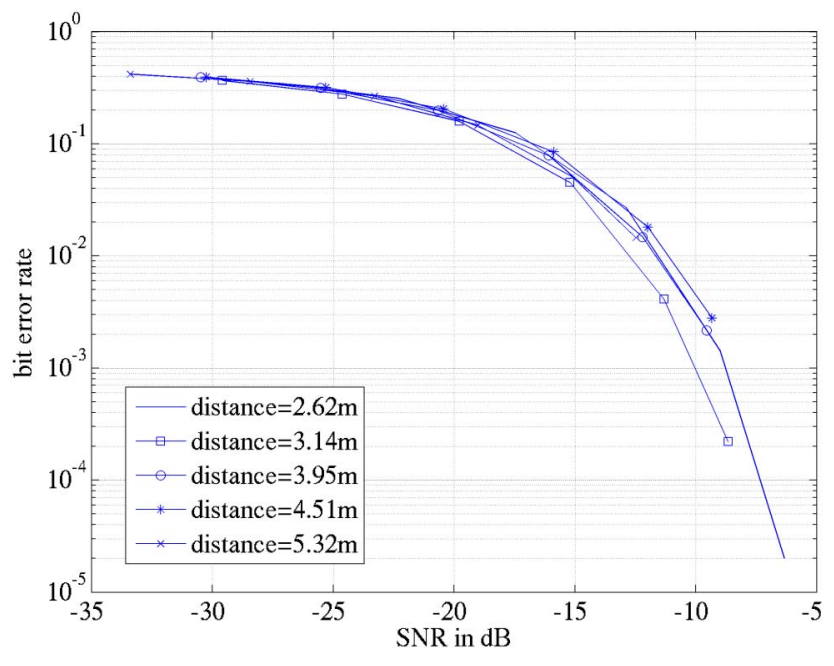

Fig. 6 Bit error rate versus signal-to-noise-ratio for a bit rate of $55.55 \mathrm{Mbit} / \mathrm{s}$

Reference [5] also investigates the impact of the bit rate on the $B E R$, however with a more simplified system model and using three pulses per bit. In [5], the $B E R$ of impulse radio transmission becomes better with reduced bit rate at given $E_{\mathrm{b}} / N_{0}$, since by reducing the bit rate, the bit duration becomes longer so that less bits are influenced by the noise.

Bit error rates of a non-ideal impulse radio system are also investigated and presented in [6] as a function of the $S N R$ and the bit rate. In contrast to the Ray Tracing approach, [6] uses only a two-path channel model, and the pulse shapes, antennas, antenna models (Butterworth filter) and the LNA are different. Instead of TH, Direct Sequence (DS) coding is used. Nevertheless, comparing [6] to the results presented here, it can be seen that the bit error rates are within a similar range, and [6] also concludes that a reduced bit rate leads to 
better $B E R$. Additionally to the explanation given above, this can also be explained by the fact that the $S N R_{\min }$ for a given $B E R$ is a function of the so-called processing gain $P G$ ([6], [7]) according to Eq. (2):

$$
P G_{d B}=10 \log \left(\frac{1}{P R F \cdot \tau_{\text {pulse }}}\right)+10 \log (N)
$$

where $\tau_{\text {pulse }}$ is the width of the pulse. When the $P R F$ is changed from $P R F_{1}$ to $P R F_{2}$ and $N$ is fixed, the difference of the processing gains is

$$
\Delta P G_{d B}=10 \log \left(\frac{1}{P R F_{1} \cdot \tau_{\text {pulse }}}\right)-10 \log \left(\frac{1}{P R F_{2} \cdot \tau_{\text {pulse }}}\right)
$$

Reducing the $P R F$ from $333 \mathrm{MHz}$ (bit rate $166.67 \mathrm{Mbit} / \mathrm{s}$, $N=2$ ) to $111.11 \mathrm{MHz}$ (bit rate $55.55 \mathrm{Mbit} / \mathrm{s}, N=2$ ) using a pulse width of $\tau_{\text {pulse }}=100 \mathrm{ps}$ leads to a theoretical $\Delta P G_{\mathrm{dB}}$ of $4.78 \mathrm{~dB}$ according to Eq. (3). Comparing the required $S N R$ values in Fig. 4 and 6 to obtain a certain $B E R$, for example a $B E R$ of $10^{-3}$, it can be seen that the difference of both $S N R$ values corresponds to the value of about $5 \mathrm{~dB}$. Fig. 7 summarizes Fig. 4 and 6 for a distance $=2.62 \mathrm{~m}$ and shows that this $S N R$ shift is always $5 \mathrm{~dB}$ and independent of the chosen $B E R$. Fig. 7 also presents the $B E R-S N R$ behavior for an increased bit rate of $500 \mathrm{Mbit} / \mathrm{s}$ (increased $P R F$ of $1 \mathrm{GHz}$, $N=2$ ). It shows that the corresponding $B E R-S N R$ curve has a kind of saturation behavior due to strong inter symbol interference (ISI), which is caused by the small pulse repetition time and the channel characteristics. The curve does not follow the typical complementary error function any more. As a consequence, Eq. (3) can only be applied for bit rates, which are small enough to avoid these saturation effects.

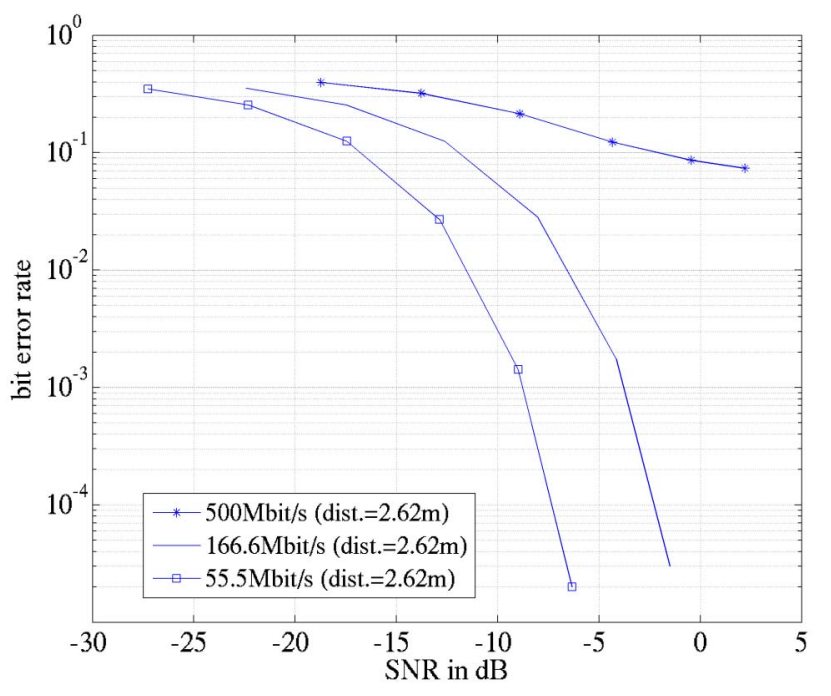

Fig. 7 Bit error rate versus signal-to-noise-ratio for three different bit rates

While in this contribution, the $P R F$ is changed to vary the bit rate, [8] varies the number of pulses per bit. In [8], the processing gain of a TH UWB system, assuming only an AWGN channel with narrowband interference, is studied. Finally, [9] investigates the processing gain based on complex processing for both, an AWGN channel and a multipath channel, however neglecting the influence of non-ideal hardware.

\section{CONCLUSIONS}

This paper evaluated the modeling of components in nonideal impulse radio systems, based on rigid assumptions. For realistic component descriptions, measurement data has been integrated. As a consequence, Dirty RF effects resulting from non-ideal hardware are included in the system model which is very important to get a realistic performance estimation of the system. The system performance was then studied in terms of bit error rate: First, it was shown how the BER degrades as a function of distance. Second, the behavior versus $S N R$ was determined. Third, the influence of the data rate was investigated, and a verification of the results by comparing the simulated processing gain with theoretical results was performed. In future, the system model will be further extended and detailed, for example by the integration of filters. A comparison of the performance between multi path and free space propagation is scheduled as well as a comparison between the Line of Sight and Non Line of Sight case.

\section{REFERENCES}

[1] J. Timmermann, D. Manteuffel, and W. Wiesbeck, "Simulation of the Impact of Antennas and Indoor Channels on UWB Transmission by Ray Tracing and Measured Antenna Patterns", IEEE International Conference on Ultra-Wideband, Singapore, Sep. 2007, pp. 194-197.

[2] G. Fettweis, M. Löhning, D. Petrovic, M. Windisch, P. Zillmann, and W. Rave, "Dirty RF - A New Paradigm", International Journal of Wireless Information Networks, vol. 14, no. 2, pp. 133-148, June 2007.

[3] J. Timmermann, M. Porebska, C. Sturm, and W. Wiesbeck, "Comparing UWB Free-space Propagation and Indoor Propagation Including Non-ideal Antennas," 10th International Conference on Electromagnetics in Advanced Applications, Sep. 2007, Torino, Italy, pp. 37-40.

[4] W. Wiesbeck et. al., "Course on Ultra Wideband Antennas", European School of Antennas, University of Karlsruhe, Germany, April 2006.

[5] S. Khatun, R. Saeed, B. Ali, M. Abdullah, C. Fun, "Ultra-Wideband (UWB) for Multimedia Applications", presented at the Auswireless 2006 Conference, 2006.

[6] S. Duenas, "Design of a DS-UWB Transceiver", M.Sc. Thesis, Royal Institute of Technology (KTH), Stockholm, Sweden, April 2005.

[7] I. Oppermann, M. Hämäläinen, J. Iinatti, "UWB Theory and Applications", John Wiley \& Sons Ltd., ISBN0-470-86917-8 (book), 2006, p. 40.

[8] J. Reed, "An Introduction to Ultra Wideband Communication Systems", Prentice Hall Communicaions Engineering and Emerging Technologies Series, ISBN 0-13-148103-7 (book), pp. 408-409, 2005.

[9] S. Rivaz, B. Denis, J. Keignart, M. Pezzin, N. Daniele, D. Morche, "Performances Analysis of a UWB Receiver using Complex Processing", IEEE Conference on Ultra-Wideband Systems and Technologies, Nov. 2003, Grenoble, France, pp. 229-233. 